\title{
Development of SOM Daily Precipitation Statistical Downscaling Model And Its Applicability Assessment in North China
}

\section{Yongdi Wang ( $\sim$ ydwang@nuist.edu.cn )}

Nanjing University of Information Science and Technology https://orcid.org/0000-0002-6716-2309

\section{Xinyu Sun}

Nanjing University of Information Science and Technology School of Electronic \& Information Engineering

\section{Research Article}

Keywords: Precipitation statistical downscaling, Self-Organizing Maps, Synoptic patterns, North China

Posted Date: October 26th, 2021

DOI: https://doi.org/10.21203/rs.3.rs-995901/v1

License: (c) (i) This work is licensed under a Creative Commons Attribution 4.0 International License.

Read Full License 


\title{
Development of SOM daily precipitation statistical downscaling model and its applicability assessment in North China
}

\author{
Yongdi Wang ${ }^{1}$, Xinyu Sun ${ }^{2}$
}

1. School of Remote Sensing \& Geomatics Engineering, Nanjing University of Information Science \& Technology, Nanjing, 210044, China

2. School of Electronic \& Information Engineering, Nanjing University of Information Science \& Technology, Nanjing, 210044, China

Abstract A statistical downscaling method based on SOM which named SOM-SD is used over North China. It's applicatibility by downscaling daily precipitation is evaluated. Indices are selected which represent the statistics of daily precipitation with regard to both precipitation amount (Prtot, SDII) and frequency (nr001), as well as extreme event (P95T, CWD, CDD). The large-scale predictors were extracted from the daily NCEP reanalysis data, while the predictand was high resolution gridded daily observed precipitation. A downscaling method based on SOM named SOMSD was presented and evaluated. In evaluating, the frequency difference of wet-dry nodes is defined. And it is confirmed that there was a significant positive correlation between frequency difference and precipitation. The SOM-SD method displayed a high skill in reproducting the climatologic statistical properties of the observed precipitation. The value of $B S$ is between 0 and $1.5 \times 10^{-4}$. Sscore is between 0.8 and 1 . The bias ranges are $-7.4 \%$ and $-11.6 \%$ for Prtot and SDII, -3.1 days for nr001, $+3.4 \%$ for P95T, -1.1 days for CWD and +3.5 days for CDD. Therefore, SOM-SD method works reasonably well.

Key words Precipitation statistical downscaling, Self-Organizing Maps, Synoptic patterns, North China

\section{Introduction}

The GCM is currently the most advanced tool available for simulating the response of the global climate system under increasing trends in greenhouse gas concentrations, but its low resolution does not meet the small-scale needs of climate impact studies. The downscaling technique can compensate the deficiency of GCM in matching spatial and temporal resolution in climate impact assessment (Burton et al. 2010; Spak et al. 2007). 
Many different statistical downscaling methods for different regions have been studied by domestic and international scholars (Linderson et al. 2004; Fowler et al. 2007; Maraun et al. 2010a, b). Among them, however, there is often the problem of underestimating the variance, which makes it less effective in the simulation of extreme climate events (Wilby et al. 2004; Hewitson and Crane 2002, 2006; Fowler et al. 2007). To address this shortcoming, Hewitson and Crane (2002, 2006) applied the SOM method to downscaling experiments (the SOM-based statistical downscaling model for precipitation, referred to as the SOM-SD model) and investigated the consistency of the model's prediction results with those of future climate downscaling. This method has overcome the shortcomings of the traditional method of underestimating the variance of the observed values to some extent (Cavazos 1999; Hewitson and Crane 2002, 2006). In recent years, many works on statistical downscaling of precipitation have been carried out in China and many results have been obtained. The statistical downscaling technique has been widely applied to the prediction of regional temperature, precipitation and other elements (Chen et al. 2003; Zhu et al. 2008; Jia et al. 2010; Wei and Huang 2010a, b). However, the applicability of the SOM-SD model in the Chinese region has not been discussed.

The application of downscaling methods to the simulation results of summer precipitation in North China, which is located at mid-latitudes, with scarce water resources and uneven intra-annual distribution of precipitation, can help to obtain refined information on future precipitation changes in the region and improve the information for future climate change scenario prediction. However, the applicability of the downscaling model needs to be tested and analyzed before the future scenario prediction.

In this study, the NCEP/NCAR reanalysis information and station precipitation information are used to apply the SOM-SD model to North China. First, forecast factors of summer precipitation are optimized by calculating Spearman's rank correlation coefficient, and then the optimized forecast factors are input into the SOM-SD model for precipitation downscaling. In order to test the effect of downscaling, the precipitation downscaling results were evaluated in terms of precipitation amount, frequency and extreme events.

\section{Data}

\section{1 Study area}

In this paper, we focus on the range of longitude from $111^{\circ}$ to $120^{\circ} \mathrm{E}$ and latitude from $36^{\circ}$ to 


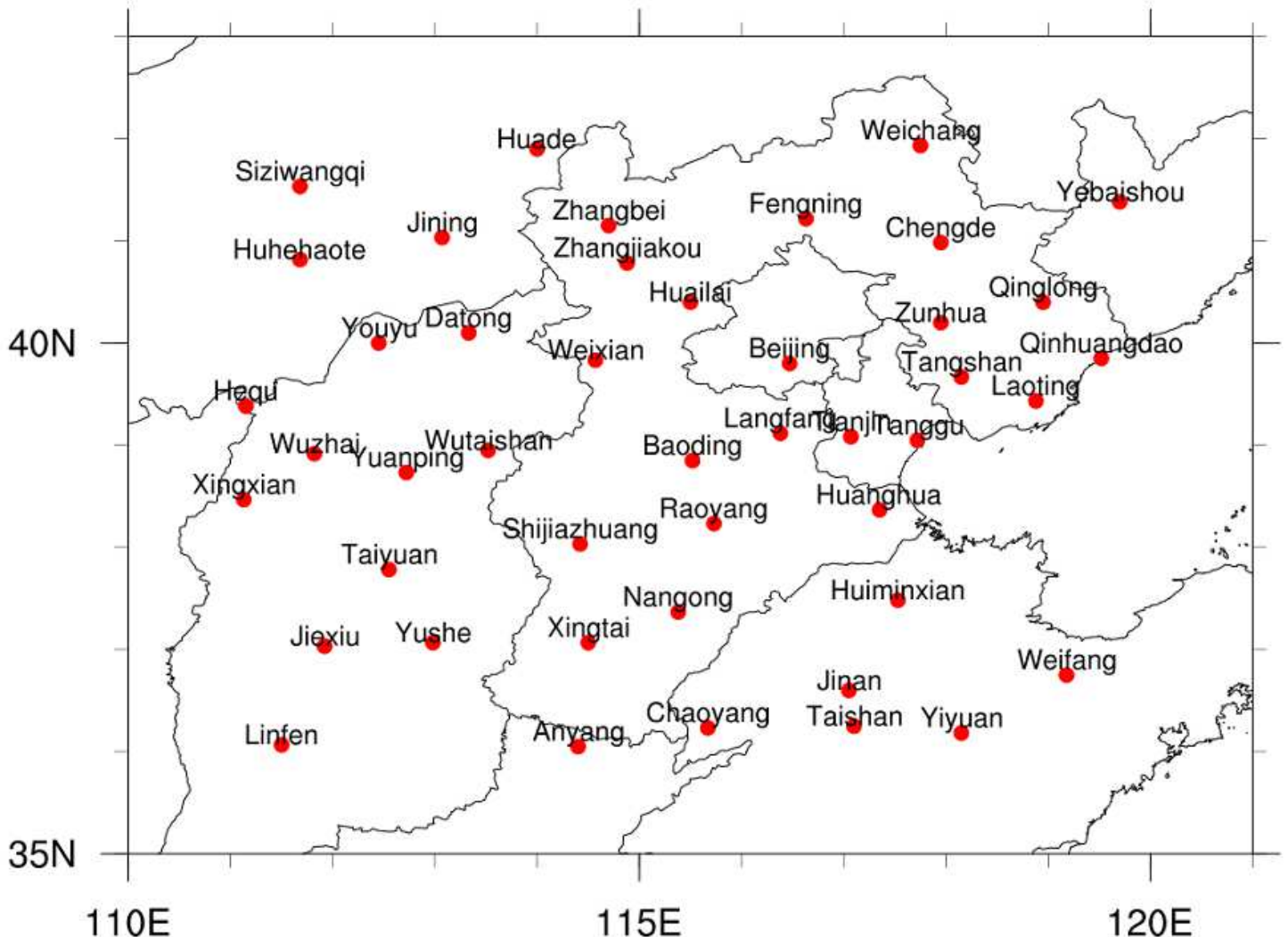

Fig. 1 Study area and station distribution

\section{2 Data}

\subsection{Station precipitation data}

The daily precipitation of the 721 weather stations in China during 1981-2010 is provided by the China Meteorological Administration. After comparison and screening, 45 stations were selected for downscaling study in this paper, whose day-by-day precipitation observations were provided by the Meteorological Information Center of China Meteorological Administration at www.cdc.gov.cn. In this paper, a 30-year time series (1981-2010) was selected among them, and $0.1 \mathrm{~mm} /$ day was used as the differentiation threshold between days with and without rain.

\subsubsection{Reanalysis data}

In the downscaling modeling, in order to obtain more accurate relationships between atmospheric circulation and ground observations, statistical downscaling models are generally built using climate element fields from reanalysis data and precipitation data from local stations first, and then the model forecast factors are projected into the built model for simulation and prediction. In the selection of climate element fields, we should consider selecting some climate variable fields 
from the reanalysis data that have obvious physical connection with local precipitation and can be simulated by the global model, so that the developed model can be used in the downscaling work of the climate model in the future. Nine climate variable fields are selected as alternative forecasters, namely: $10 \mathrm{~m}$ surface wind field (uas, vas), $500 \mathrm{hPa}$ wind field (ua500, va500), $850 \mathrm{hPa}$ relative humidity (hur850) and specific humidity (hus850), $10 \mathrm{~m}$ surface air temperature (tas), $850 \mathrm{hPa}$ to $500 \mathrm{hPa}$ vertical decreasing rate of temperature Lapse rate, and sea level pressure (slp). There are obvious physical links between these forecast factors and local precipitation, among which, the wind field at different heights can characterize the lower atmospheric convergence and upper atmospheric dispersion, the humidity field and surface temperature field can characterize the water vapor condition and its saturation degree, the Lapse rate of temperature vertical decrement from $850 \mathrm{hPa}$ to $500 \mathrm{hPa}$ can characterize the degree of atmospheric instability stratification, and the sea level pressure field can characterize the large scale The sea level pressure field can characterize the largescale circulation situation. These data are reanalyzed by NCEP/NCAR on a daily basis (resolution $2.5^{\circ} \times 2.5^{\circ}$ ), and then the fields of these variables are considered together as comprehensive elements describing the atmospheric state, and their correlation with precipitation at the station is calculated and judged as a condition for selection as a forecast factor. After this selection, the forecasting factor of precipitation at the target station can be used for SOM training.

\subsection{Division of time periods}

The first 20 years (1981-2000) were used for the establishment of the downscaled precipitation model, referred to as the downscaled model rate period, and the last 10 years (2001-2010) were used for the testing of the downscaled precipitation model, referred to as the downscaled model testing period.

\section{3 Data pre-processing}

In order to be able to reflect the atmospheric state around the target station, the data can be preprocessed according to the method proposed by Ning et al. (2012) for the grid information before downscaling: for each station the $\mathrm{n}$ variable fields around the station can be described by $n 1 \times 19$ vectors (Fig. 2). 


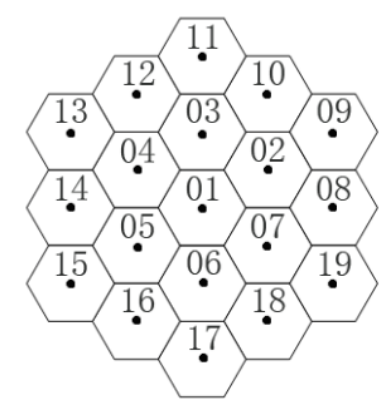

Fig. 2 Variable field interpolation (19 hexagons selected around the station)

As shown in Fig. 2, point 01 is the location of the station, and a square hexagon with side length of $1^{\circ}$ is made at the center of this point, and this square hexagon is extended outward in two circles. For each hexagon, 6 vertices can form 6 triangles, and the value of each triangle vertex can be obtained by weighting the values of the 4 grid points around the vertex according to the distance and calculating the 6 triangle centers of gravity respectively, and then the value of the center of each hexagon is obtained by averaging the values of the 6 centers of gravity. The center of each hexagon represents the interpolation result of a climate variable in the region, and there are 19 points including the center point 01 . The data of these 19 points can form a $1 \times 19$ vector, which represents a new variable field describing the conditions around the station. The day-by-day data of all climate variable fields in a given time range are divided into seasons to form the input data for SOM training in the corresponding season.

\section{4 Normalization of data}

The magnitudes of the different climate variable fields are not uniform, and each variable needs to be normalized before SOM training. For a variable $X$ at a given grid point $(i, j)$, the normalization process is given by

$$
\hat{X}_{i j}(t)=\frac{X_{i j}(t)-\mu}{\sigma}
$$

where $\hat{X}_{i j}(t)$ is the result of normalizing the original variables $X_{i j}(t)$ at grid point $(i, j)$ at time $t$. and $\mu$ and $\sigma$ denote the mean and standard deviation of the sample data, respectively.

\section{Methods}

\section{1 Principles and methods for the selection of predictive factors}

The selection of predictors is an important part of the downscaling process, and the usability of the downscaling results depends on the appropriateness of the selection of predictors. The 
selection of predictors is very complicated, for example, some predictors are very important but may have low explanatory power for the forecast objects, and the explanatory power of each predictor may change with time and space (Wilby et al. 2004). The selection of forecast factors needs to consider the following points (Wang et al. 2012; Liu 2013).

(a) There should be a strong correlation between the predictor and the precipitation, and some direct or indirect physical connection.

(b) Not only the forecast factor should be successfully simulated by the climate model, but also the link between the forecast factor and precipitation should be successfully simulated by the model.

(c) It is necessary to pay attention to the uniformity of the data, and the forecast factors should be selected from the common factors in the reanalysis data and models, which can avoid the prediction errors caused by the errors between observations and simulations to a certain extent.

The Spearman rank correlation coefficient method is used in this paper. The reason for adopting this method is that the daily precipitation time series do not satisfy the assumption of normality of linear methods in previous studies due to their characteristics of non-negativity, many zero values, and non-normality. In contrast, various types of statistical forecasting models and statistical test methods as well as the commonly used Pearson correlation coefficient calculation require the information to conform to a normal distribution. Common solutions are power transformation (cube root or quadratic root, etc.) normalization, hyperbolic tangent transformation normalization; setting certain thresholds to eliminate negative downscaling results, etc. However, these practices lack a physical basis (Yang et al. 2009) and some precipitation series still do not obey normal distribution after such treatment due to the high number of zero values.

In this case, the Spearman rank correlation coefficient can be considered for forecaster preference. This correlation coefficient has the advantages of not requiring the data to obey a normal distribution, good robustness, and insensitivity to outliers. When the data series do not obey a normal distribution, have poor quality, or are suspected to be affected by outliers, it is more reasonable to use the rank correlation coefficient compared to the Pearson correlation coefficient.

For any two variables $x_{i}$ and $y_{i}$, the samples are $n$, and $x_{i}$ and $y_{i}$ are ordered by their respective sizes, and the respective ordinal numbers are noted as $D_{x}^{i}$ and $D_{y}^{i}$, then the ordinary correlation coefficient between the ordinal numbers is the Spearman rank correlation coefficient 
(noted as $r_{s}$ ).

Since equation (3) is simpler, equation (3) is generally used in the actual analysis.

The significance of the rank correlation coefficient can be tested by the $Z$ statistic.

$$
Z=\frac{r_{s}}{\sqrt{1 /(n-1)}}
$$

\section{2 The main implementation steps of the SOM-SD mode1}

The SOM-SD model consists of three main steps: First, the SOM technique requires training of the preferred forecasters to identify all possible weather patterns around the target station. Second, the observed precipitation sequences are grouped into different sets of precipitation values according to the category number of the clustering results, and each set of precipitation values corresponds to a weather pattern, thus establishing the association between the forecasters and the precipitation. Third, the forecast factors of each day are projected into the SOM nodes obtained from the training, and the precipitation values are randomly selected from the set of precipitation values corresponding to the class according to the category number of the projection result. The following is a detailed description of the three main steps of downscaling for one station, and the rest of the stations in the study area adopt the same process to realize their own downscaling.

\subsubsection{Obtain various weather patterns representing the atmospheric state around}

\section{the station through SOM clustering}

The SOM training is performed on the preferred forecast factors of 19 grid points around the station to obtain a set of ordered two-dimensional nodes, each of which can represent a weather pattern. The daily multivariate climate variable field is then mapped to one of the SOM nodes to identify the category of weather patterns around the target site each day. Among them, the number of nodes is an optional value, and the number of SOM nodes selected varies among studies in 

2006), which is somewhat subjective and requires attention when determining the number of nodes: if the number of nodes is too small it will On the contrary, if the number of nodes is too large, the number of samples assigned to each node is too small, which is not conducive to the estimation of precipitation downscaling values in the later period. However, after repeated tests and comparisons, it is found that the number of nodes does not have a significant effect on the downscaling effect. In this paper, we do not analyze in depth the degree of influence of the change of SOM node size on the downscaling results, and only take the SOM output node size of $4 \times 4$ as an example for analysis.

\subsubsection{Establishing the relationship between station precipitation and weather}

\section{patterns around the station}

After the SOM training is completed, the relationship between station precipitation and weather patterns can be established. The time period used for model establishment can be called the rate period of the model, and the time period used for model testing can be called the testing period of the model. For each day of the model rate period, its forecast factor can be compared as a sample with the nodes obtained from SOM training (calculating the Euclidean distance between them), and the one with the shortest Euclidean distance can be selected based on the calculation of the Euclidean distance, i.e., the winning node. Then, the precipitation value corresponding to that day is projected to that winning node, and that precipitation value can be assigned to a class according to the serial number of the winning node. The above process is repeated until all precipitation values are classified, so that the precipitation values are also classified into corresponding groups according to the SOM nodes, and each group of precipitation values corresponds to one SOM node.

Each precipitation value is related to the corresponding weather pattern (SOM node) in this way. Because each node represents a different atmospheric state, the precipitation probability density curves plotted with the set of precipitation values corresponding to each node also vary, i.e., the probabilities of precipitation values that can be produced by different nature of weather patterns are different, and this correspondence between precipitation values and weather patterns can be well captured by projection. In this paper, we use the gamma distribution in each category of precipitation values to fit the precipitation probability density function to obtain the fitted parameters of the probability density function for each category, and then use the fitted parameters of the obtained probability density function to generate random precipitation values in the next step of the 
downscaling process.

\subsection{Obtaining downscaled precipitation series based on Monte Carlo simulation}

By projection, the relationship between precipitation values and weather modalities is established. Next, the validation data of the model testing period can be used to evaluate the downscaled model simulation results. For each downscaled target station, its forecast factors are compared with the trained SOM nodes one by one, and the corresponding node class number of each day can be obtained, through which the forecast factors of each day are related to the corresponding set of precipitation values. Then, the corresponding precipitation value for a particular day can be generated from a certain set of precipitation values by random resampling, which is generated according to the parameters of the fitted probability density function. Precipitation values for all days of that time period are generated in this way, and a precipitation downscaling time series can be formed.

(a) Prtot (mm/season)

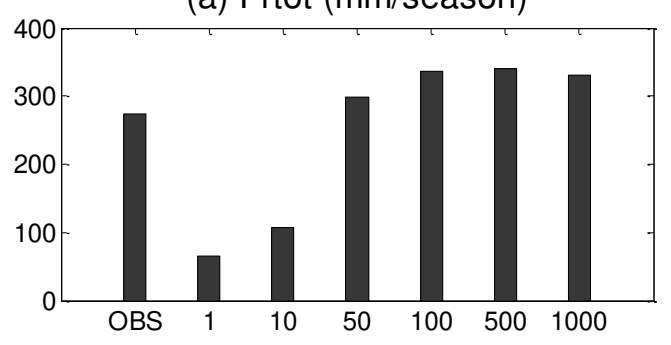

(c) nr001 (days)

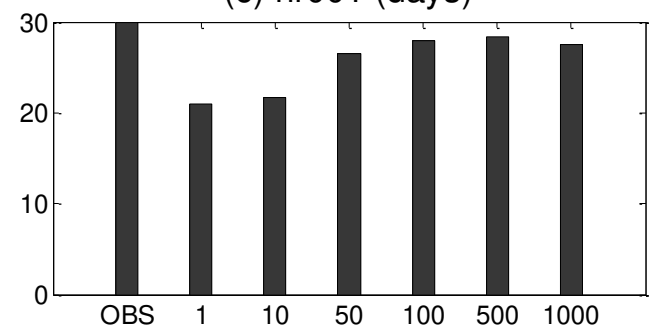

(b) SDII (mm/day)

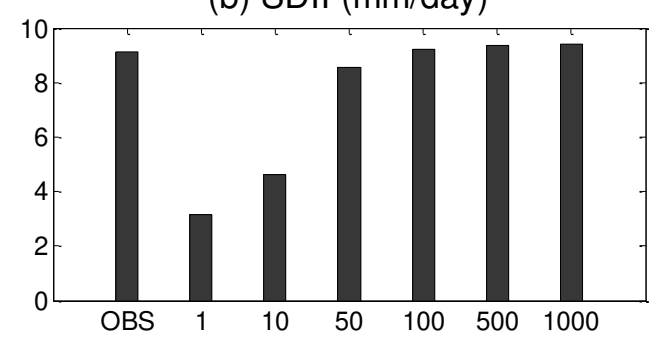

(d) P95T (percent)

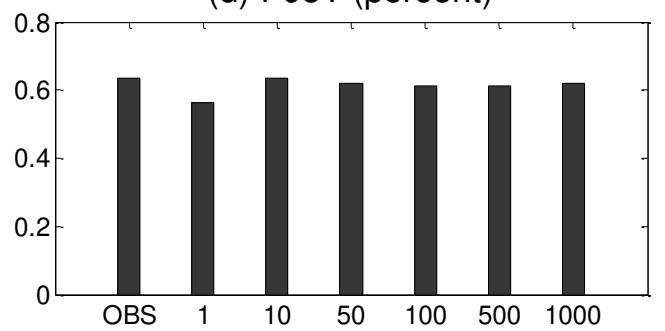

Fig. 3 Multiple repetition sampling experiments for descending scale sequences (number of samples: $1,10,50,100,500,1000)$

In the random generation of the precipitation downscale sequences, the random resampling of the downscale results was repeatedly tested and compared several times in order to be able to reflect the probability distribution characteristics of the precipitation downscale database to the maximum extent. Each generated sequence of equal length to the observed value sequence is considered as a sample in the precipitation database. To test whether the downscaled results gradually stabilize with increasing sample size, several different metrics (Prtot, SDII, nr001, P95T, etc.) can be used. The 
results are plotted here for 1, 10, 50, 100, 500, and 1000 times (see Fig. 3).

In Fig. 3, as the number of resampling increases, each indicator value gradually converges with the observed value results and gradually stabilizes. As the number of resampling reaches 1000 times, the above time series are repeated 1000 times to obtain 1000 simulated series of precipitation downscaling of equal length with the station observation series of precipitation.

\section{3 Methodology for evaluating the simulation capability of the SOM-SD mode1}

\section{3. 1 Evaluation index of the degree of error of the probability density function}

In order to quantitatively analyze the simulation performance of the SOM-SD model for the probability density function (PDF) of precipitation at each station, the Brier Score ( $B S$; Brier 1950) and the Significance Score ( $S_{\text {score }}$; Perkins et al. 2007; Watterson 2008) based on the probability density function can be used to evaluate the error level of the simulated and observed probability density functions. (Perkins et al. 2007; Watterson 2008) to assess the degree of error between the simulated and observed probability density functions (Fu et al. 2013).

$$
\begin{aligned}
B S & =\frac{1}{n} \sum_{i=1}^{n}\left(P_{m i}-P_{o i}\right)^{2} \\
S_{\text {score }} & =\sum_{i=1}^{n} \operatorname{Minimum}\left(P_{m i}, P_{o i}\right)
\end{aligned}
$$

where $n$ denotes the probability density function divided into $n$ equal length intervals, and $P_{m i}$ and $P_{o i}$ are the values of the probability density functions of the observed and simulated variables in the $i$ interval, respectively. $B S$ is a measure of the non-coincidence between the observed and simulated probability density functions, and the smaller the non-coincidence, the closer the value of the index is to zero, which means the better the simulation result. $S_{\text {score }}$ reflects the area of the mutual overlap between the simulated and observed probability density functions of the two series. The larger the overlapping area is, the larger the value is, and it reaches the maximum value of 1 when they completely overlap, so the closer the value of this indicator is to 1 , the better the simulation result is.

3.3.2 Evaluation index of statistical characteristics of daily precipitation statistical downscaling results 
In order to observe the statistical characteristics of the daily precipitation statistical downscaling results, some commonly used precipitation indices are selected in this paper (the specific meaning of each indicator is detailed in Table 1), and most of these indicators are selected from the core indicators of the EU STARDEX program for analyzing extreme climate events (http://www.cru.uea.ac.uk/cru/projects/stardex/). They mainly include the following: precipitation amount (Prtot), precipitation intensity (SDII), number of rainy days (nr001), extreme precipitation contribution (P95T), maximum consecutive days with rain (CWD), and maximum consecutive days without rain $(\mathrm{CDD})$.

Table. 1 Definition of precipitation index

\begin{tabular}{clc}
\hline Acronym & \multicolumn{1}{c}{ Definition } & Unit \\
\hline Prtot & Total season precipitation & $\mathrm{mm} /$ season \\
SDII & Simple daily intensity (mean daily precipitation on wet days) & $\mathrm{mm} / \mathrm{day}$ \\
nr001 & Mean number of rainy days for daily precipitation $\geq 0.1 \mathrm{~mm}$ & Days \\
P95T & Percentage of rainfall from events beyond $95^{\text {th }}$ percentile value of overall precipitation & Percent \\
CWD & Maximum consecutive wet days & Days \\
CDD & Maximum consecutive dry days & Days \\
\hline
\end{tabular}

\section{Application of SOM-SD model in North China}

The SOM downscaling method was applied to North China to test its applicability.

\section{1 Selection of prediction factors}

For each station, each alternative predictor (hur850, hus850, slp, Lapse tate, tas, uas, vas, ua500, va500) is interpolated into 19 grid points around the station by a bilinear interpolation method (as shown in Fig. 2). In this way, each predictor can form a time series at each grid point separately (19 $\times 9=171$ time series in total for the 9 predictor fields). The Spearman correlation coefficients are then obtained for each of these predictor time series with the station precipitation time series and tested for significance. The correlation coefficient is useful to measure the degree of correlation between two factors in an objective way. The results are plotted in Fig. 4 for the Beijing station as an example. 


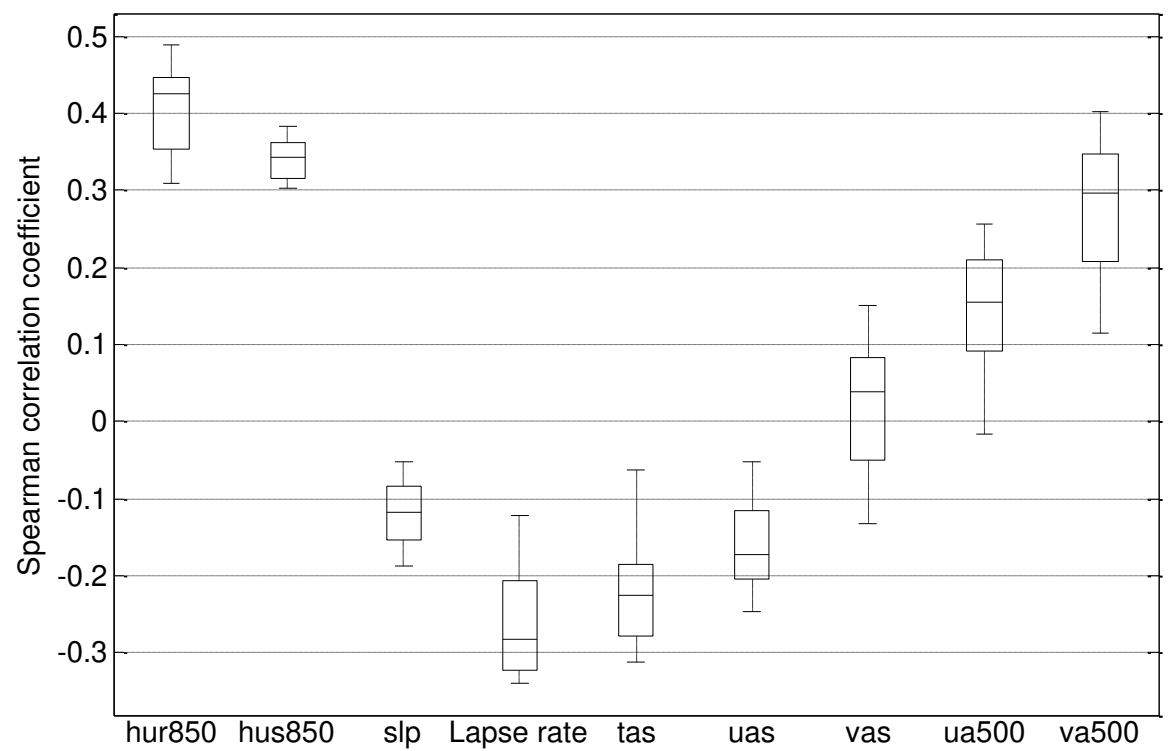

Fig. 4 Forecast factor preference results of summer precipitation downscaling model for Beijing station

In Fig. 4, the horizontal axis shows the nine predictors and the vertical axis shows the Spearman correlation coefficients of each predictor with the precipitation series $R$. From the figure, we can see that the predictors hur850, hus 850 , ua500 and va500 have a significant positive correlation with precipitation (at the 95\% confidence level). The remaining forecast factors, except vas, all have negative correlations with precipitation (slp, lapse rate, tas, and uas). The above results indicate that in Beijing, the low-level humidity field is more closely related to summer precipitation and can provide sufficient water vapor supply, which is one of the key factors affecting summer precipitation. The smaller the vertical decreasing rate of temperature the more unstable the atmosphere is, and the more obvious the vertical motion is, which is also more closely related to summer precipitation. The lower the air pressure around the station, the more favorable the formation of precipitation. The trend of surface air temperature and precipitation is also inverse, as the solar radiation absorbed by the ground decreases in rainy weather in summer, and the surface air temperature decreases accordingly. 

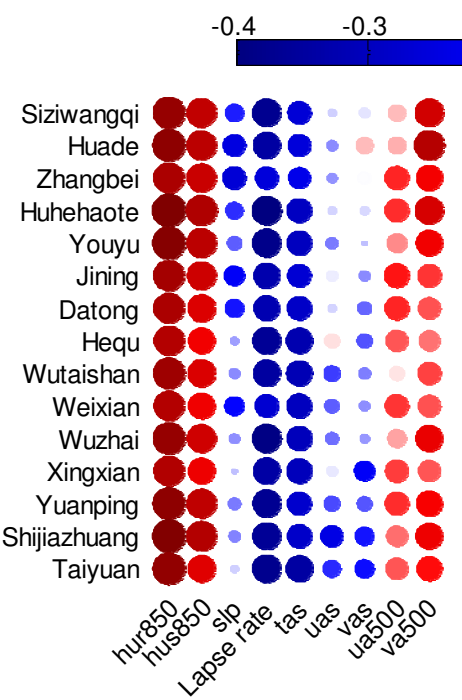
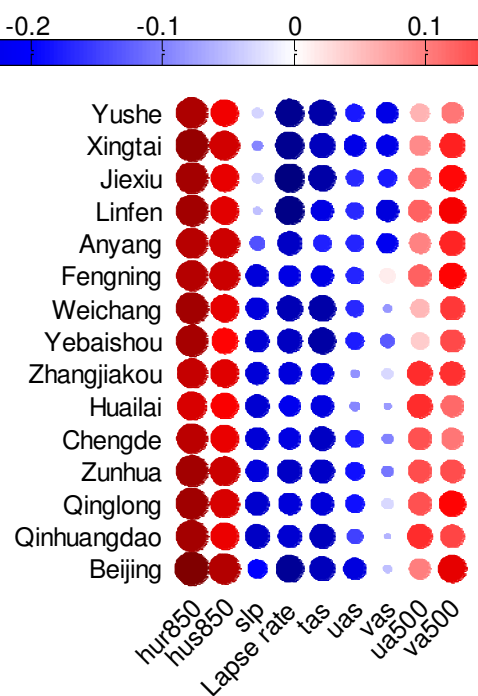

$\begin{array}{lll}0.2 & 0.3 & 0.4\end{array}$
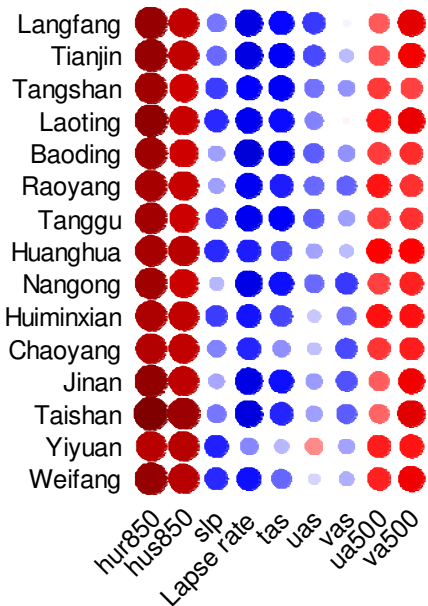

Fig. 5 Calculation results of correlation coefficients between forecast factors and precipitation series at various stations in North China

The Spearman correlation coefficients are calculated for the remaining stations according to the same calculation method, and Fig. 5 shows the results of the correlation coefficients. In Fig. 5, red color indicates positive correlation and blue color indicates negative correlation. From Fig. 5, we can see that forecast factors such as hur 850 , hus 850 , ua500 and va500 are significantly positively correlated with precipitation at most of the stations, while forecast factors such as lapse rate and tas are significantly negatively correlated with precipitation at most of the stations. The above results show that for different meteorological conditions, the effect of slp, uas and vas on precipitation is relatively weak.

The above results indicate that the relationship between each meteorological element and summer precipitation varies somewhat for different meteorological stations. Before the downscaling, it is necessary to optimize the forecast factors for different stations, so as to better reflect the atmospheric conditions around the stations.

\section{2 Analysis of precipitation characteristics at different nodes of SOM}

\subsection{Cumulative probability distribution function $\mathrm{CDF}$ curves of precipitation} values corresponding to SOM nodes

Fig. 6 shows the CDF curves of the cumulative probability distribution of daily precipitation corresponding to the $16 \mathrm{SOM}$ nodes. The cumulative probability distribution curves of precipitation from node 1 to node 16 show a strong regularity: there is a clear pattern of gradually decreasing precipitation along the diagonal line (from the upper left to the lower right). For the nodes 
corresponding to the atmospheric states favorable to precipitation formation (e.g., nodes 1, 2, 5, and 6), the corresponding precipitation CDFs show larger precipitation values. On the contrary, for the nodes corresponding to unfavorable precipitation generation (e.g., the 11th, 12th, 15th, and 16th nodes), the corresponding precipitation CDFs show smaller or close to zero precipitation values. The above results show that there are significant differences in the CDF curves in different SOM nodes. This implies that important differences in different precipitation conditions have been accounted for in the clustering of weather patterns using the SOM method. In the context of weatherscale circulation, this difference in the CDF curves in different SOM nodes has some physical implications, i.e., a correspondence between precipitation and circulation fields has been established.
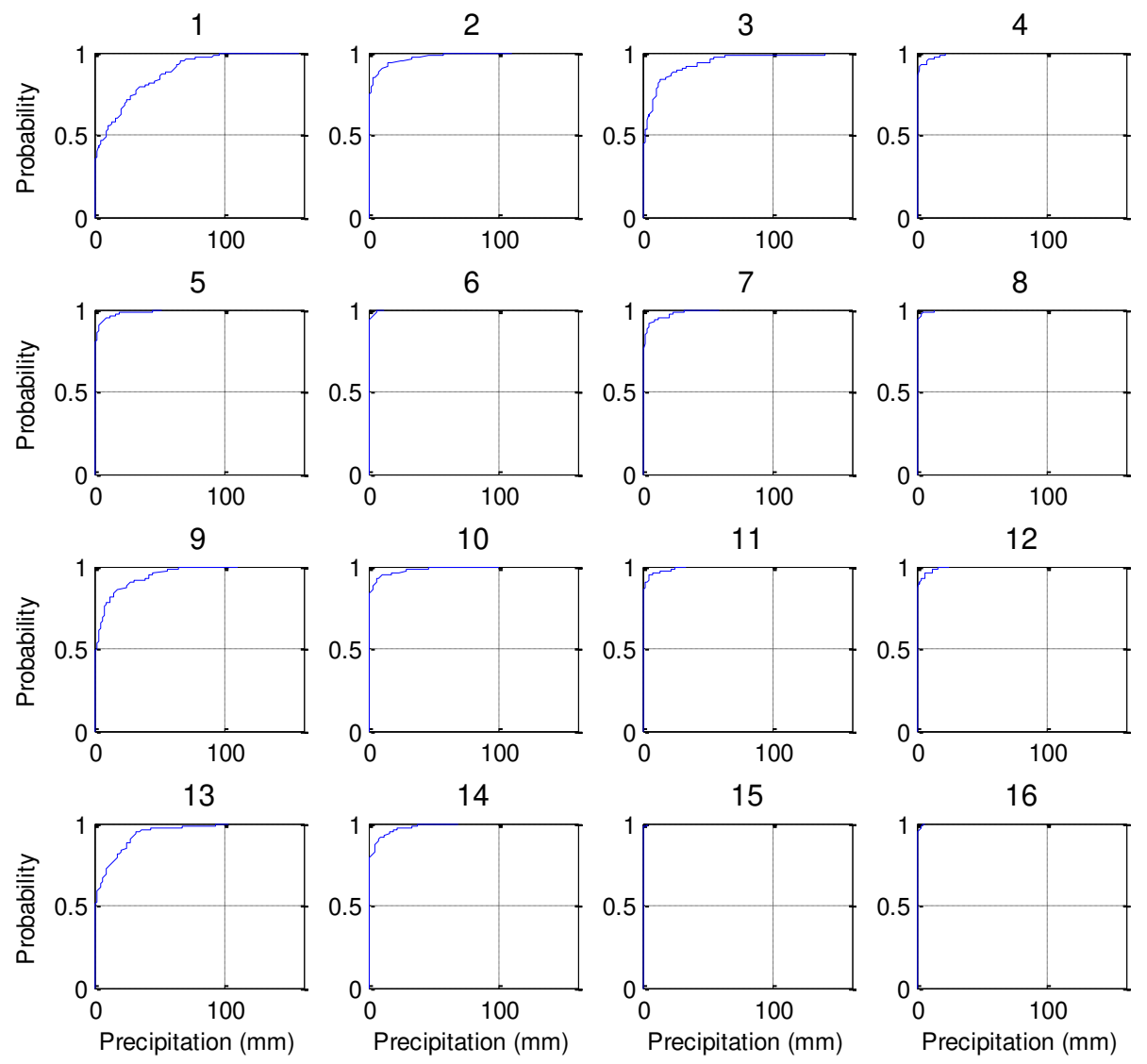

Fig. 6 Cumulative probability distribution function (CDF) of precipitation for the $16 \mathrm{SOM}$ nodes corresponding to the training results

In order to observe the circulation fields corresponding to the 16 SOM nodes and analyze their distribution patterns, the node distribution of each forecast factor can be plotted separately for analysis. Considering the complexity of the causes of summer precipitation, in addition to small and medium rainfall, the more important summer precipitation is heavy rainfall due to strong convective weather, while most of the heavy rainfall is not caused by atmospheric circulation anomalies, and 
the dominant factors inducing summer rainstorms should be humidity and temperature, which tend to cause atmospheric instability and thus favor the formation of precipitation. The distance level fields of two representative variables, $850 \mathrm{hPa}$ relative humidity (hur850) and $850 \mathrm{hPa}$ to $500 \mathrm{hPa}$ vertical decreasing rate of temperature (Lapse rate), are plotted below for analysis (see Figs. 7 to 8). Fig. 7 shows the node distribution of the $850 \mathrm{hPa}$ relative humidity (hur850) pitch level field. In the figure, there is a positive pitch level center of relative humidity in the top left corner of nodes (1), (2), (5), and (6) that exactly surrounds Beijing station, which corresponds to the wet node in Fig. 6. And the nodes (11), (12), (15) and (16) in the lower right corner all have a negative pitch level center of relative humidity exactly around Beijing station. This corresponds to the dry node in Fig. 6. Fig. 7 is more consistent with the distribution pattern of the nodes in Fig. 6, indicating that $850 \mathrm{hPa}$ relative humidity is an important factor affecting precipitation and needs to be considered when downscaling. In addition to the relative humidity field, the specific humidity field (not shown) also shows a similar pattern, so the specific humidity is also an important factor affecting summer precipitation and should also be selected when performing variable preferences.

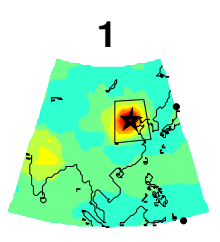

5

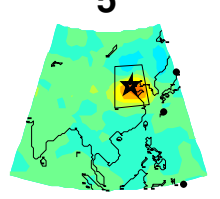

9

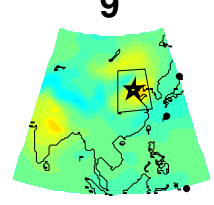

13

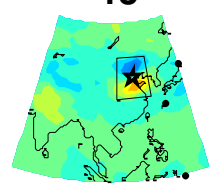

2

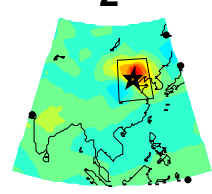

6

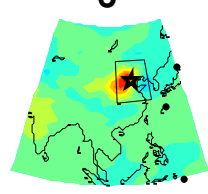

10

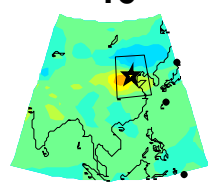

14

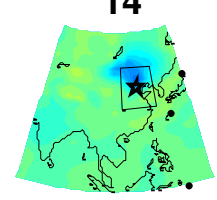

3

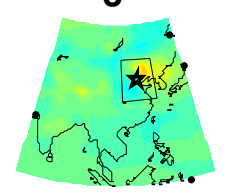

7

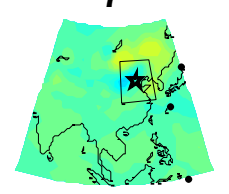

11

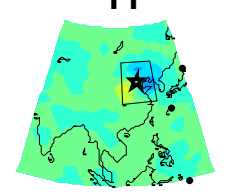

15

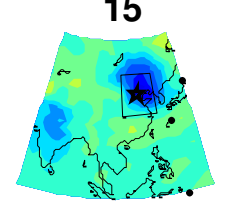

4

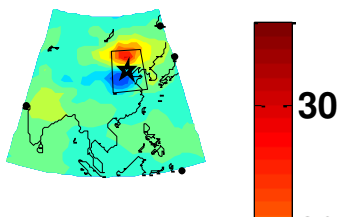

8

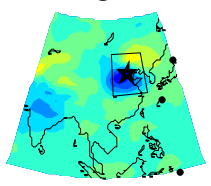

12

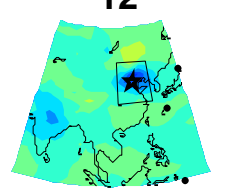

16

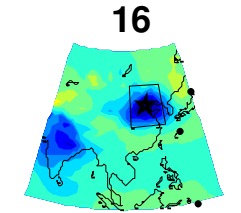

20

10

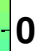

$-10$

$-20$

$-30$

Fig. 7 Distribution of $850 \mathrm{hPa}$ relative humidity field (\%) for the $16 \mathrm{SOM}$ nodes corresponding to the training results 


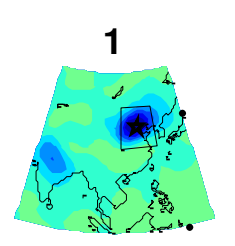

5

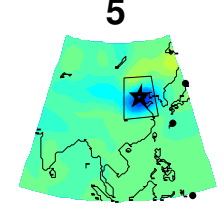

9

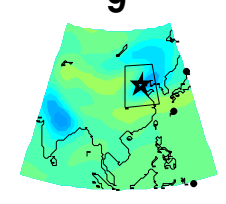

13

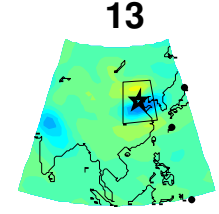

2

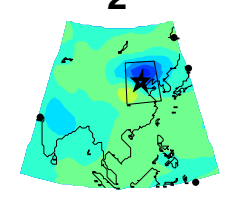

6

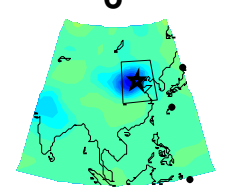

10

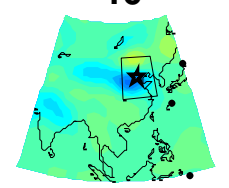

14

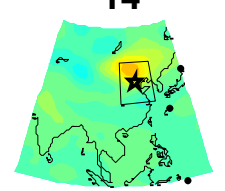

3

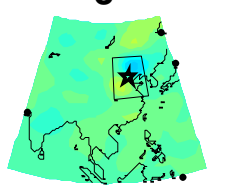

7

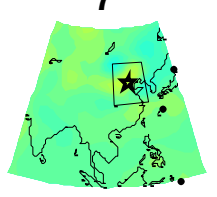

11

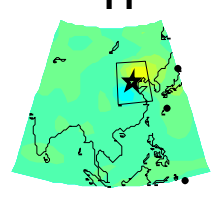

15

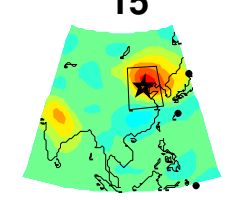

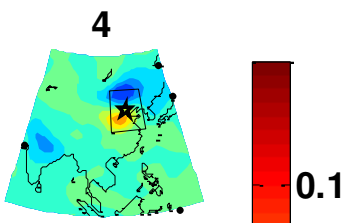

8

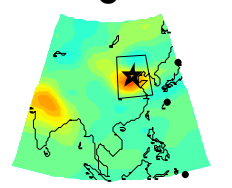

12

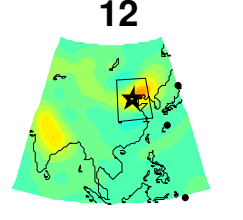

16

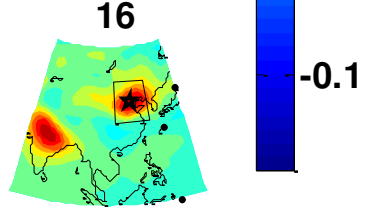

0.05

0

$-0.05$

$-0.1$

Fig. 8 Distribution of vertical decreasing rate of temperature $\left({ }^{\circ} \mathrm{C} / \mathrm{hm}\right)$ for the $16 \mathrm{SOM}$ nodes corresponding to the training results

In Fig. 8, the node distribution of the vertical decreasing rate (Lapse rate) of temperature from $850 \mathrm{hPa}$ to $500 \mathrm{hPa}$ is plotted in the distance level field. The distribution also has a more obvious distribution pattern from the upper left corner to the lower right corner. In the upper-left corner, the negative pitch level surrounds the Beijing area, which corresponds to the node with large values of precipitation (i.e., wet node), and in the lower-right corner, the positive pitch level surrounds the Beijing area, which corresponds to the node with small values of precipitation (i.e., dry node). It is shown that the vertical decreasing rate of temperature (Lapse rate) from $850 \mathrm{hPa}$ to $500 \mathrm{hPa}$ is also an important factor affecting the summer precipitation.

To observe the relationship between the circulation field and station precipitation, the sea level pressure (slp) distance level field is plotted in Fig. 9. If slp is closely related to precipitation, the arrangement of SOM nodes should have the following pattern: nodes that are relatively close to each other and nodes that are opposite in nature are arranged far away from each other. For the slp field, the node in the upper left corner (1) should be low-pressure dominant (indicated in blue), while the node in the lower right corner (16) should be high-pressure dominant (indicated in red), and the rest of the nodes gradually transition from high to low. However, the results of the slp field 
shown in Fig. 9 do not fully follow this pattern. It indicates that among the influencing factors of summer precipitation, the slp field is not the most closely related to precipitation. It also shows that in some cases where high pressure prevails, precipitation may also be brought due to atmospheric instability, and in some cases where low pressure prevails, precipitation may not be formed due to lack of water vapor. Therefore, the causes of summer precipitation cannot only consider the circulation field, but need to consider more meteorological elements together.

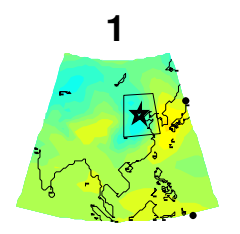

5

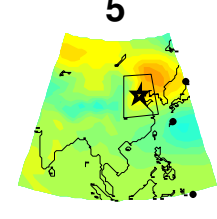

9

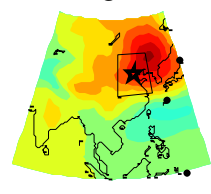

13

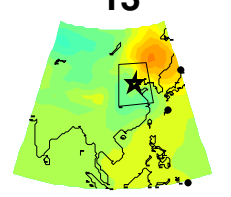

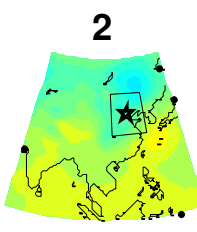

6

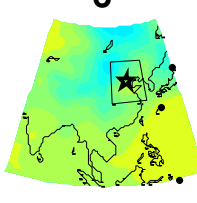

10

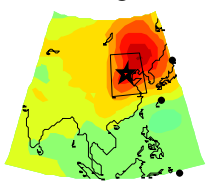

14

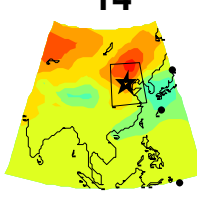

3

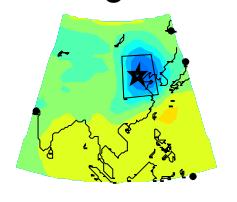

7

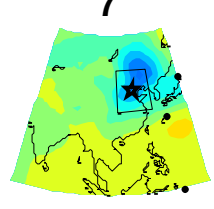

11

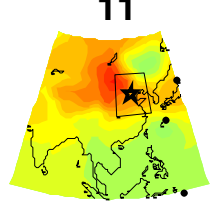

15

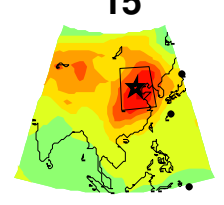

4

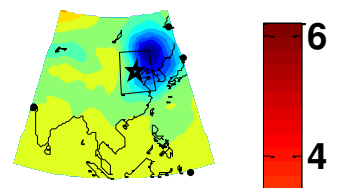

8

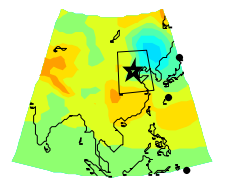

12

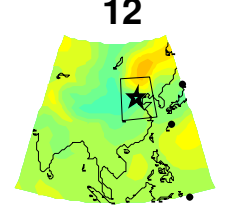

16

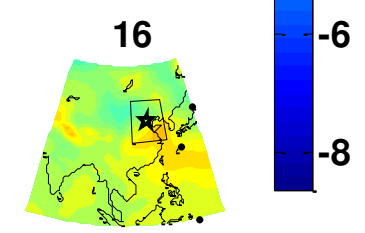

Fig. 9 Distribution of sea level pressure field (hPa) for $16 \mathrm{SOM}$ nodes corresponding to the training results

Which of these nodes are more likely to form precipitation needs further analysis and validation. The number of rainy days, total precipitation, average daily precipitation and 95th percentile precipitation values of precipitation in each node were counted separately, and the results are plotted in Fig. 10.

From Fig. 10, it can be seen that the 1st node has 101.0days of rain, $132.8 \mathrm{~mm}$ of annual average total precipitation, $20.1 \mathrm{~mm} /$ day of daily average precipitation, and $68.1 \mathrm{~mm}$ of 95 th percentile precipitation value, and the above indicators (frequency, total, average, and extreme) show the maximum value among all nodes many times, so it can be defined as a wet node. On the contrary, the 15th node shows less precipitation in each of the above indicators and can be defined 
as a dry node. Other than this the rest of the nodes can be considered as transition nodes.

(a) number of rainy days (days)

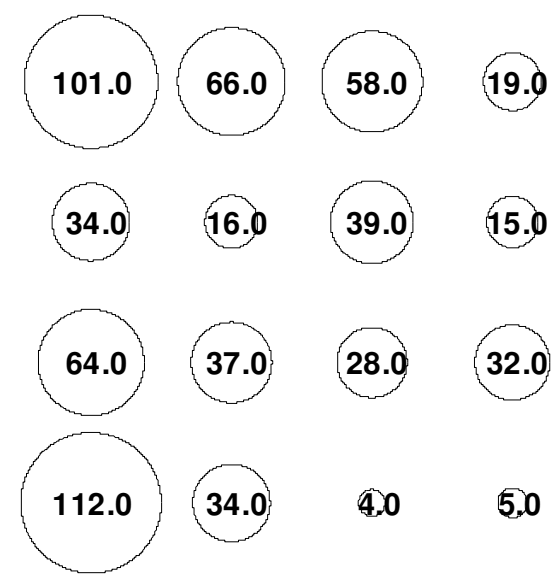

(c) daily average precipitation ( $\mathrm{mm} /$ day)
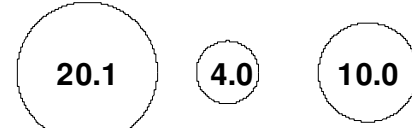

1.1

2.0

0.4

2.4

1.4

1.0

9.23 .0

0.1

0.2 (b) total annual precipitation (mm)

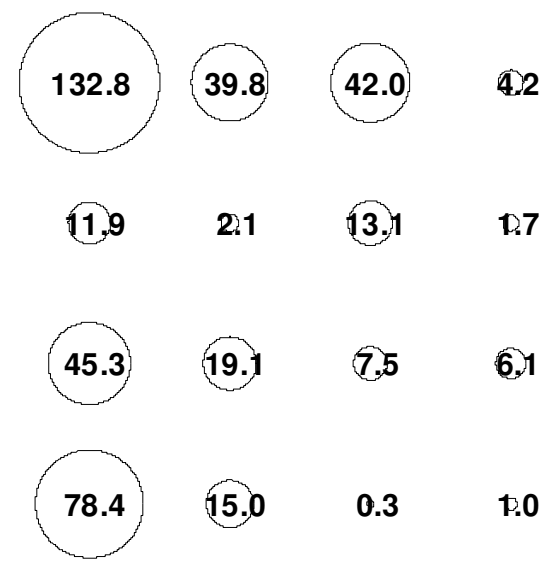

(d) 95th percentile precipitation value $(\mathrm{mm})$

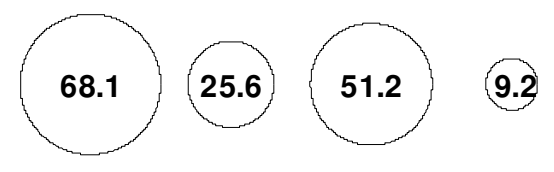

$\begin{array}{llll}10.7 & 2.5 & 14.8 & 07\end{array}$

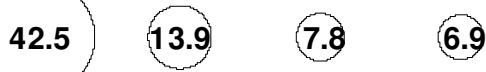

$\begin{array}{llll}34.3 & 18.2 & 0.2 & 1.8\end{array}$

Fig. 10 Quantitative determination of the indicators of dry and wet nodes. Indicator performance on each node: (a) number of rainy days (days), (b) total annual precipitation ( $\mathrm{mm}$ ), (c) daily average precipitation (mm/day), (d) 95th percentile precipitation value (mm)

The significant difference between the CDF curves of the dry and wet SOM nodes indicates that the SOM clustering results of atmospheric states can, to a certain extent, distinguish different precipitation scenarios at different weather scales (different atmospheric states bring different levels of precipitation). This result fully demonstrates the advantages of SOM clustering: (a) when there are many forecast factors and the relationship with precipitation is non-linear, the clustering can be successfully achieved by using SOM. (b) SOM can cluster different atmospheric states into nodes of different nature, and different nodes bring different magnitudes of precipitation. (c) The results of clustering are continuous in the whole, and the evolution of different nodes can be easily observed 
(b) slp (hPa)

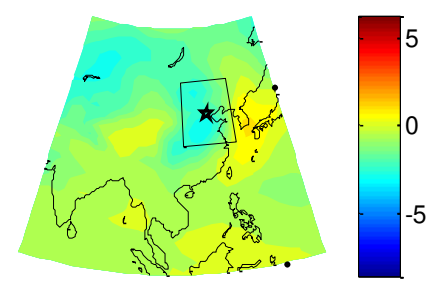

(e) $\operatorname{sip}(\mathrm{hPa})$ (d) hur850 (\%)
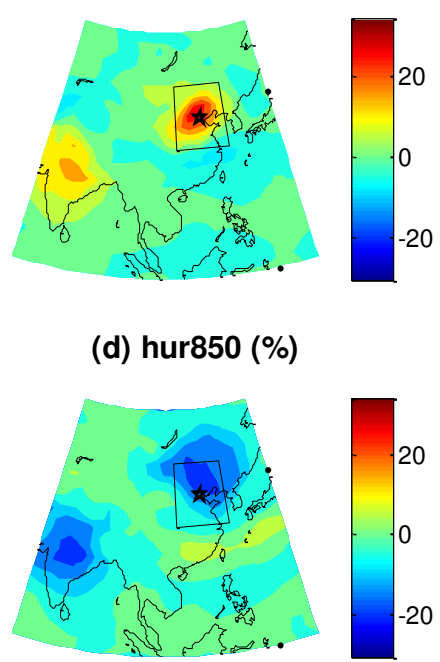

413

Fig. 11 Comparison of the distribution states of each predictor in the wet and dry nodes at the Beijing station (where a-c are the distance level fields of each predictor in the wet nodes and d-f are the distance level fields of each predictor in the dry nodes)

In Fig. 11, the distribution states of each predictor in the wet and dry nodes are compared, where Fig. 11a-c show the spatial distribution states of the distance level field of each predictor in the wet nodes. It can be seen that in the wet node (Fig. 11, row 1), the $850 \mathrm{hPa}$ relative humidity (hur850) is at a positive distance level (Fig. 11a), which is significantly positively correlated with precipitation, while the $850 \mathrm{hPa}$ to $500 \mathrm{hPa}$ vertical decreasing rate (Lapse rate) is at a low value (Fig. 

variables have a good relationship with precipitation. In this state, sufficient water vapor can be provided to Beijing station, which is conducive to the formation of precipitation. The state of each variable in this node near Beijing Station is the most likely state to bring precipitation, and the possibility of bringing more precipitation is high. In the dry node (Fig. 11, row 2), the $850 \mathrm{hPa}$ relative humidity (hur850) is basically close to or at a negative pitch level around Beijing (Fig. 11d), while the vertical decreasing rate of temperature (Lapse rate) from $850 \mathrm{hPa}$ to $500 \mathrm{hPa}$ is basically close to a high value (Fig. 11f). It cannot provide sufficient water vapor source for Beijing stations, which is not conducive to precipitation formation.

The 500hPa wind field distance level is plotted in Fig. 12, where Fig. 12a shows the 500hPa wind field distance level in the wet node and Fig. 12b shows the 500hPa wind field distance level in the dry node. In Fig. 12a, the 500hPa wind field pitch level rotates clockwise with the Korean Peninsula as the center, and the wet airflow turns northward after landing westward in the eastern coastal area of China, which can bring sufficient water vapor to the Beijing area. In Fig. 12b, the $500 \mathrm{hPa}$ wind field level has a central point near Mongolia, and the wind field level rotates clockwise around this point. The dry airflow near Beijing is mainly from the north, and this airflow cannot bring the necessary water vapor for precipitation when it goes south through Beijing, so there are no precipitation conditions.

(a) $500 \mathrm{hPa}$ wind anomalies for wet $\operatorname{node}(\mathrm{m} / \mathrm{s})$

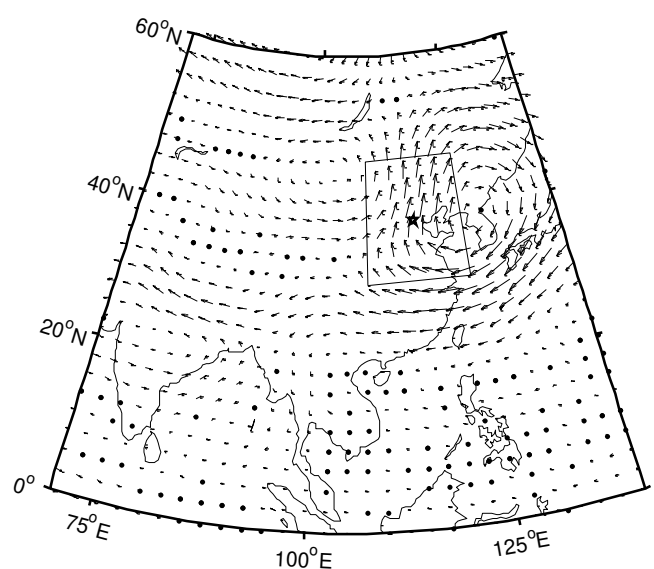

(b) $500 \mathrm{hPa}$ wind anomalies for $\mathrm{dry} \operatorname{node}(\mathrm{m} / \mathrm{s})$

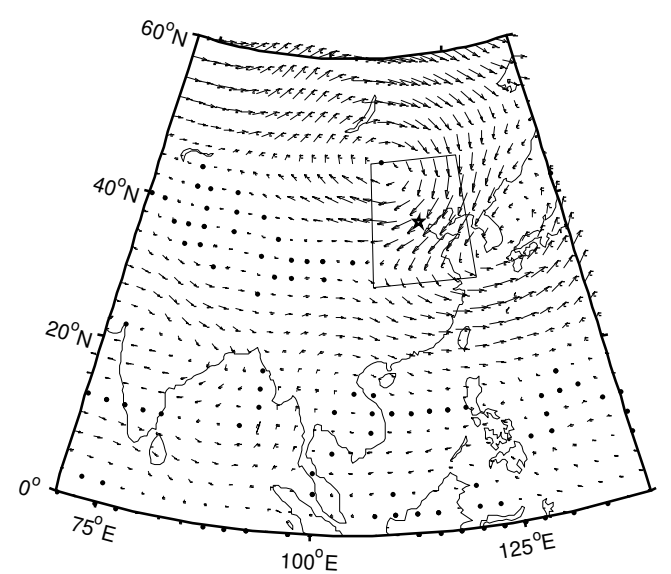

Fig. 12 Comparison of the distribution of $500 \mathrm{hPa}$ wind field level in the wet and dry nodes at Beijing station (where a is the $500 \mathrm{hPa}$ wind field level in the wet node and $\mathrm{b}$ is the $500 \mathrm{hPa}$ wind field level in the dry node) 
The above analysis shows that several forecast factors, such as $850 \mathrm{hPa}$ relative humidity (hur850), $850 \mathrm{hPa}$ to $500 \mathrm{hPa}$ vertical decreasing rate (Lapse rate) and $850 \mathrm{hPa}$ specific humidity (hus850) (not shown), 10m surface air temperature (tas) (not shown), 500hPa wind field (ua500, va500), are the main forecast factors affecting precipitation in the dry and wet nodes, and because the states of each forecast factor in the dry and wet nodes are diametrically opposed, thus bringing different precipitation.

4.2.3 Analysis of the relationship between interannual variation of wet and dry node frequency difference and interannual variation of average daily precipitation

The trend of its frequency difference (wet node frequency minus dry node frequency) will better reflect the change of precipitation compared to the trend of wet node or dry node frequency. Because if the frequency difference increases upward, it already contains the following situations: the wet node frequency remains unchanged and the dry node frequency decreases; the wet node frequency increases and the dry node frequency remains unchanged; the wet node frequency increases while the dry node frequency decreases; it increases in the same direction and the wet nodes at Beijing station does affect the change of precipitation. In order to test the universality of

In order to test the reasonableness of the above hypothesis, the year-by-year dry and wet nodal frequency differences at each node of Beijing station were counted, and the summer average daily precipitation at the station was also counted year by year. The calculated results show the following pattern: The frequency difference between the wet and dry nodes also shows a similar trend, and the correlation coefficient between them reaches 0.54 (at the $95 \%$ confidence level).

The above results indicate that the change of the frequency difference between wet and dry this law in the rest of the stations in North China, similar calculations were performed for the rest of the stations, and the average correlation coefficient of the 45 stations was 0.41 (at the $95 \%$ 
confidence level), indicating the universality of this law. There is a significant positive correlation between the interannual variation of the difference between the occurrence frequency of the wet and dry nodes and the interannual variation of precipitation. The trend of precipitation can be estimated from the variation of dry and wet nodes.

\section{3 Simulation test of SOM-SD mode1 in North China}

The previous analysis has demonstrated that weather variability can be captured using the SOM method, which portrays the atmospheric state represented by SOM nodes, and different SOM nodes correspond to different precipitation characteristics, i.e., the CDF curves of precipitation are not consistent at different SOM nodes. In addition, it is possible that different precipitation amounts may be brought at the same node (with similar atmospheric conditions). To capture this randomness, random repetitive sampling can be performed from the CDF curve of each node to ensure that the downscaling results can match the statistical characteristics of the observed value series. In addition, the reasonableness of the dry and wet node definitions and the forecast factor preferences have been tested in the previous work. Next, it is necessary to check whether the trained SOM can be used to generate a precipitation day-by-day time series, and whether the time series has a high agreement with the observed value series in terms of magnitude, frequency and other characteristics, which can be judged by the evaluation of the precipitation downscaling results. Assessment of the effect of precipitation downscaling requires examination of the statistical characteristics of the precipitation downscaling results in terms of precipitation quantity, precipitation frequency, and extreme precipitation.

\subsubsection{Analysis of simulation results on the probability density function of} downscaling results

In order to observe whether the downscaled results can well reflect the probability density characteristics of the observed values, the Q-Q plots of the downscaled results of summer precipitation are plotted in Fig. 13 (taking Beijing, Tianjin, Shijiazhuang, Hohhot, Taiyuan and Jinan stations as examples). In Fig. 13, the scatter points of precipitation basically fall on the diagonal line, indicating that the two obey a uniform distribution. The above results show that the downscaling results of the SOM-SD model can well reflect the probability distribution characteristics of summer precipitation in North China. 
(a) Beijing

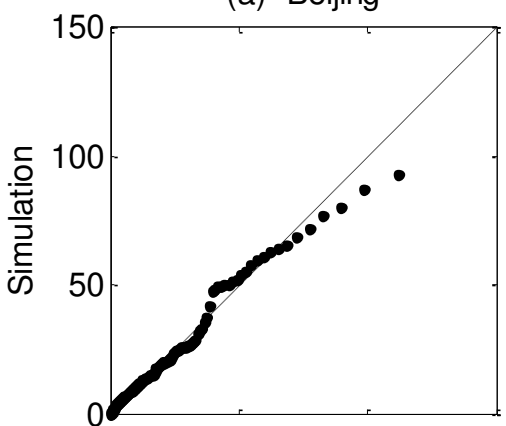

(d) Huhehaote

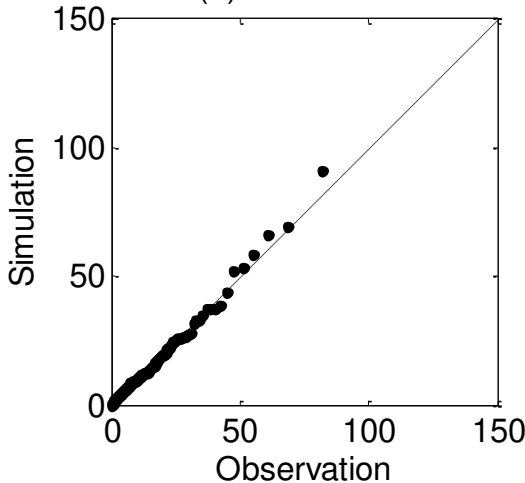

(b) Tianjin

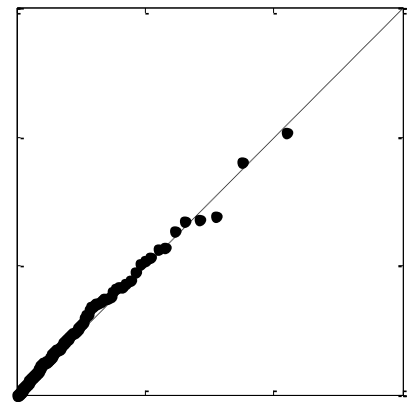

(e) Taiyuan

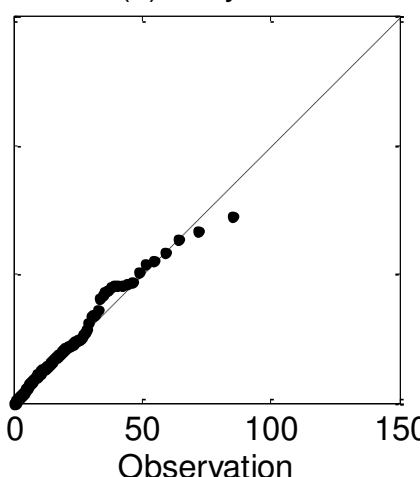

(c) Shijiazhuang

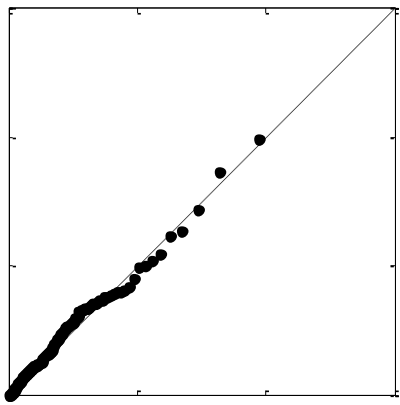

(f) Jinan

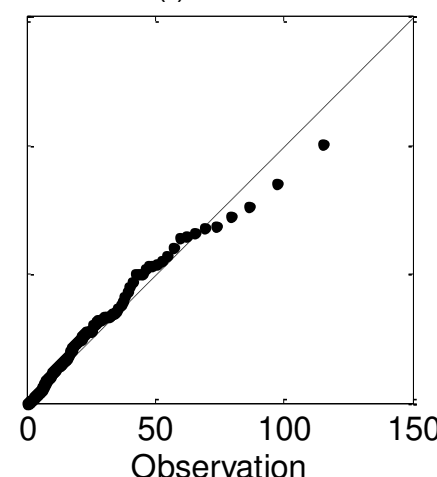

Fig. 13 Q-Q plots of summer precipitation downscaling results (with stations in Beijing, Tianjin, Shijiazhuang, Hohhot, Taiyuan and Jinan as examples; the horizontal axis is the precipitation observation and the vertical axis is the precipitation downscaling results)

In order to quantitatively evaluate the ability of the downscaling model to simulate the probability density function of precipitation, two indicators, $B S$ and $S_{\text {score }}$, are used to evaluate the errors between the downscaled simulation results and the observed values at each station, and the results are plotted in Fig. 14. The closer the value of the indicator $B S$ is to 0 and the closer the value of the indicator $S_{\text {score }}$ is to 1, the better the simulation capability is. From Fig. 14, the values of the indicators $B S$ are in the range of 0 to $1.5 \times 10^{-4}$ and the values of the indicators $S_{\text {score }}$ are in the range of 0.8 to 1 , indicating that the SOM-SD model can simulate the probability density function of summer precipitation in North China well. 

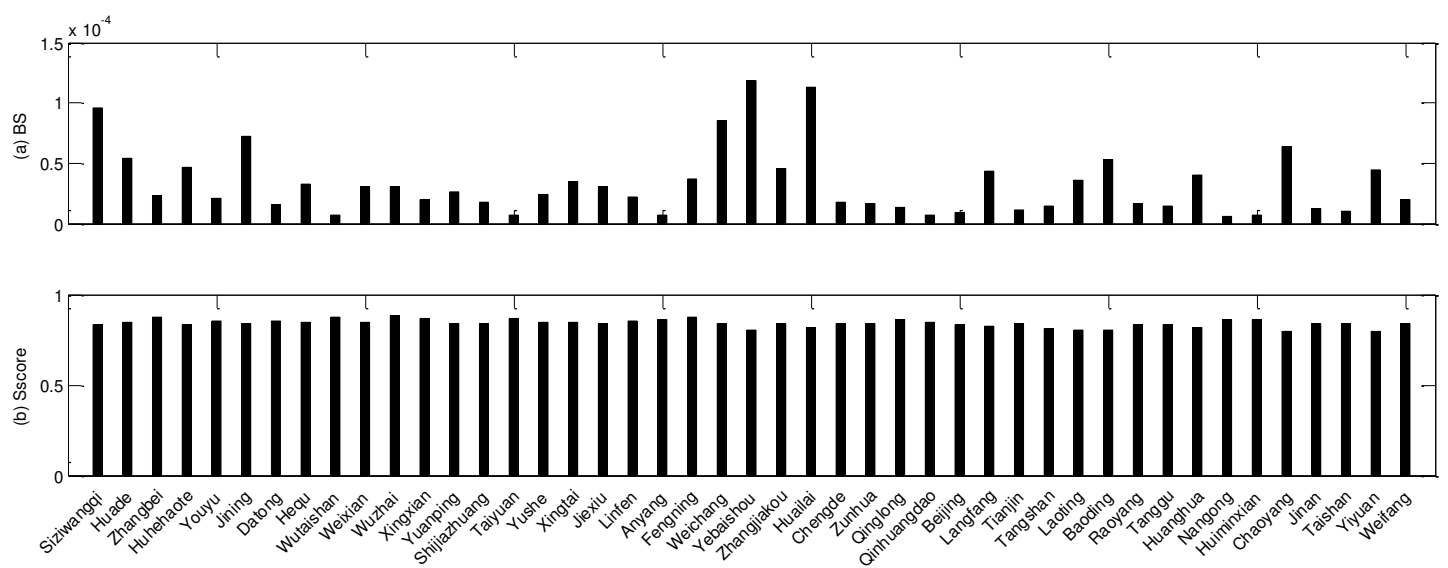

Fig. 14 Quantitative evaluation of the summer precipitation downscaling probability density function pdf simulation results for each station in North China: (a) histogram of $B S$ indicators for each station, (b) histogram of $S_{\text {score }}$ indicators for each station

4.3.2 Evaluation of the validity of the SOM-SD simulation results for station precipitation

In order to evaluate the applicability of the model for summer precipitation in North China more quantitatively, the total precipitation (Prtot), precipitation intensity (SDII), total number of rainy days (nr001), extreme precipitation contribution (P95T), maximum continuous rainy days (CWD), and maximum continuous rainless days (CDD) are used to evaluate the precipitation, precipitation frequency, and extreme precipitation, respectively. The simulation error (simulated value - observed value) and the error percentage [(simulated value - observed value)/observed value $\times 100 \%$ ] need to be calculated. The observed and simulated values were first taken as regional averages at 45 stations, and then the simulated errors and error percentages were calculated, and the comparison of the downscaled results with the observed values is listed in Table 2.

Table. 2 Comparison of precipitation downscaling results with observed values (observations and simulations were averaged at 45 stations, respectively, and then the errors were calculated)

\begin{tabular}{cccccc}
\hline Acronym & Unit & Observation & Downscaling & Simulation error & Error percentage \\
\hline Prtot & mm/season & 300.5 & 287.3 & -13.2 & $-4.4 \%$ \\
SDII & mm/day & 9.5 & 8.5 & -1.0 & $-10.5 \%$ \\
nr001 & days & 31.8 & 28.7 & -3.1 & $-9.7 \%$ \\
P95T & percent & $56.9 \%$ & $58.3 \%$ & $1.4 \%$ & $2.5 \%$ \\
\hline
\end{tabular}




\begin{tabular}{cccccc}
\hline CWD & days & 5.1 & 4.1 & -1.0 & $-19.6 \%$ \\
CDD & days & 11.1 & 14.6 & 3.5 & $31.5 \%$ \\
\hline
\end{tabular}

As can be seen from Table 2, the average value of the total annual summer precipitation Prtot observations for 45 stations in North China is $300.5 \mathrm{~mm}$, and the downscaled simulation result is $287.3 \mathrm{~mm}$ (underestimated by $13.2 \mathrm{~mm}$ ), with an error percentage of $-4.4 \%$. The observed value of precipitation intensity SDII is $9.5 \mathrm{~mm} /$ day, and the downscaled simulation result is $8.5 \mathrm{~mm} /$ day (underestimated by $1.0 \mathrm{~mm} /$ day), with an error percentage of $-10.5 \%$. The number of rainy days nr001 is 31.8 days, and the downscaled simulation result is 28.7 days (underestimated by 3.1 days), with an error percentage of $-9.7 \%$. The observed extreme precipitation contribution rate $\mathrm{P} 95 \mathrm{~T}$ is $56.9 \%$, and the downscaled simulation result is $58.3 \%$ (overestimated by $1.4 \%$ ), with an error percentage of $2.5 \%$. The maximum continuous rainfall day CWD observation is 5.1 days, and the downscaled simulation result is 4.1 days (underestimated by 1.0 days), with an error percentage of $-19.6 \%$. The maximum continuous rain-free day is 11.1 days, and the downscaled simulation result is 14.6 days (overestimated by 3.5 days), with an error percentage of $31.5 \%$.

For each station, the downscaled simulation errors have obvious spatial distribution differences. The spatial distribution of the simulation errors is plotted below for each station (Fig. 15a-f).

(a) Evaluation of precipitation simulations

The observed values of total precipitation (Prtot) in summer and the error percentages of the downscaled results are plotted in Fig. 15a. The downscaled simulation errors of each station are positive and negative, with the average size of $25.3 \mathrm{~mm}$ and the average error percentage of total precipitation Prtot of each station is $-7.4 \%$, which indicates that the SOM-SD model has a strong ability to simulate total precipitation (Prtot). 
(a) Relative error (Prtot: \%)

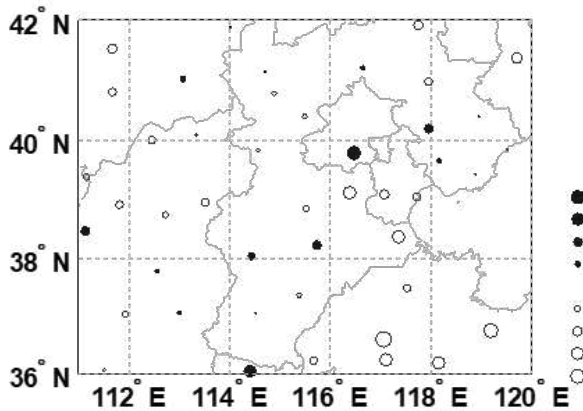

(c) Relative error (nr001: \%)

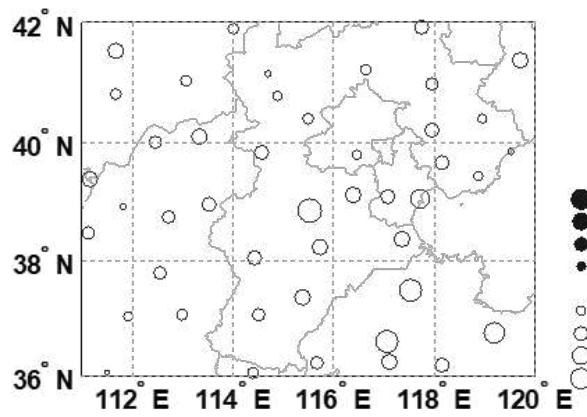

(e) Relative error (CWD: \%)

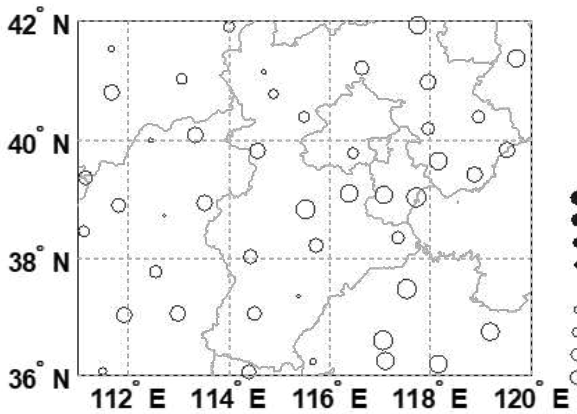

(b) Relative error (SDII: \%)

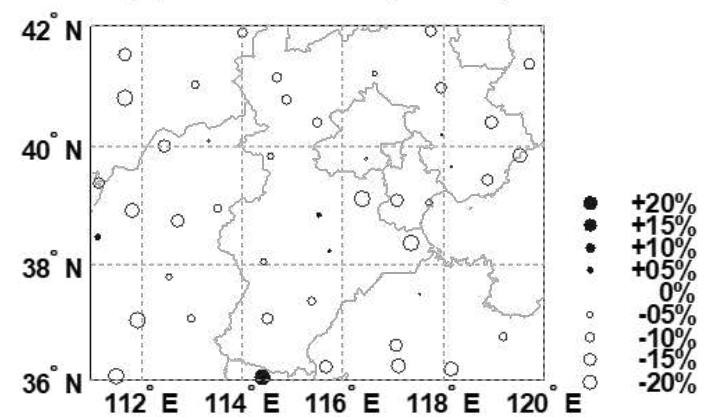

(d) Relative error (P95T: \%)

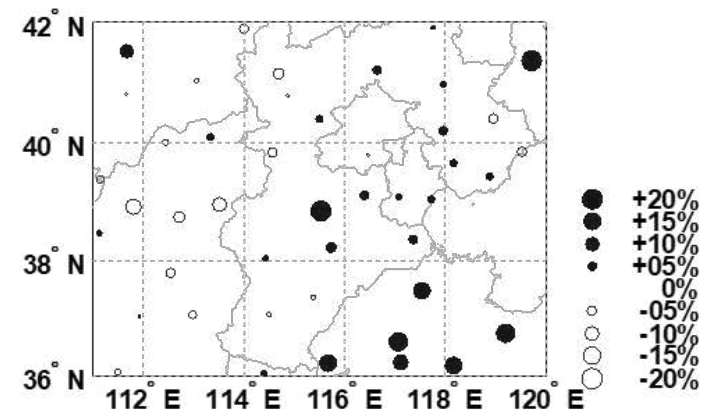

(f) Relative error (CDD: \%)

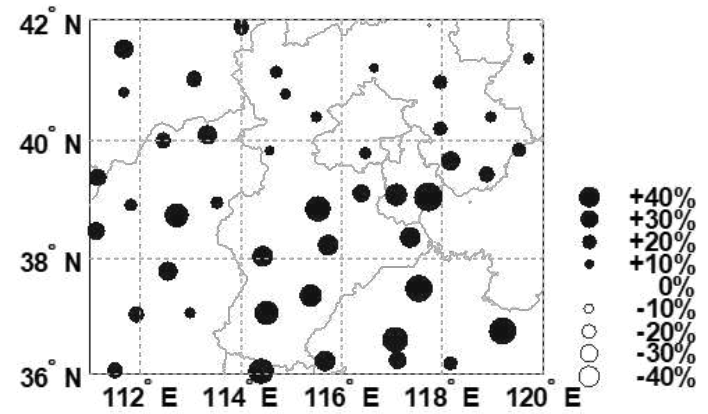

Fig. 15 Precipitation indicators: (a) Prtot; (b) SDII;

; (c) nr001; (d) P95T; (e) CWD; (f) CDD

The spatial distribution of the simulation error and error percentage of the precipitation intensity SDII relative to the station precipitation observations are plotted in Fig. 15b. The simulation error is negative at most stations with a mean value of $-1.1 \mathrm{~mm} /$ day, and the mean SDII error percentage is $-11.6 \%$. This indicates that the downscaling model underestimates the precipitation intensity at most stations.

(b) Evaluation of precipitation frequency simulations

In addition to precipitation amount, another important indicator is precipitation frequency. The frequency characterization of precipitation downscaling results is measured by the number of precipitation days above $0.1 \mathrm{~mm}$ (nr001). Fig. 15c gives the comparison between the downscaled simulation results and the observed values for the nr001 index. 
(nr001) in the downscaled results is underestimated compared to the observed values (error percentage $-9.8 \%)$ at all stations.

(c) Assessment of extreme precipitation

The biggest challenge when downscaling precipitation using global climate models is the reproduction of extreme precipitation events, which often have much larger economic and social impacts than the monthly average precipitation. Therefore, finally, the ability of downscaling models to simulate extreme precipitation events needs to be assessed. Here, two sets of metrics are used: the first set is the extreme precipitation contribution P95T, which represents the percentage of precipitation above the 95th percentile of all precipitation (Fig. 15d). The second group is CWD and CDD, where CWD denotes the maximum consecutive days with rain (Fig. 15e) and CDD denotes the maximum consecutive days without rain (Fig. 15f).

In Fig. 15d, the contribution of precipitation above the 95th percentile (P95T) is indicated, which has a simulation error of $+3.4 \%$ and an error percentage of $+6.1 \%$, with a relatively good agreement between the downscaled simulation results and the observed values. It indicates that the SOM-SD model has a strong ability to simulate extreme summer precipitation events in North China. The CWD on the maximum continuous rainfall day (Fig. 15e) is generally underestimated at all stations (average underestimation 1.1days, error percentage -20.0\%). The CDD (Fig. 15f) is generally overestimated (mean overestimation 3.5 days, error percentage $+31.1 \%$ ).

\section{Conclusion}

This paper presents a statistical precipitation downscaling model based on self-organizing mapping, the SOM-SD model, which is applied to the simulation of summer precipitation in North China and its applicability is evaluated and tested. The evaluation work uses total precipitation (Prtot), precipitation intensity (SDII), total number of rainy days (nr001), extreme precipitation contribution (P95T), maximum continuous rainy days (CWD), and maximum continuous rainless days (CDD) to evaluate and analyze the simulation results of summer precipitation at 45 stations in North China in terms of precipitation amount, precipitation frequency, and extreme precipitation. The main conclusions drawn are as follows.

(a) The representative local atmospheric states around the stations that are closely associated with the formation of precipitation can be described jointly by a set of climate element fields combined together. A certain atmospheric state can be automatically identified and successfully 
captured using the SOM-based circulation mode typing method, and they are automatically classified into different categories (i.e., weather patterns), each of which corresponds to a different precipitation probability distribution characteristic.

(b) There is a very significant positive correlation between the interannual variation of the difference in the frequency of wet and dry nodes and the interannual variation of precipitation. It is not necessary for the model to simulate the frequency of each climate mode well, as long as the trend of the difference in the frequency of the wet and dry nodes can be successfully simulated, the trend of precipitation can be estimated. This can provide a new perspective to determine the credibility of the predicted future climate change results.

(c) The selection of forecast factors is a critical aspect of precipitation downscaling, which directly affects the downscaling results. When downscaling precipitation at different stations, it is necessary to select forecast factors for each station separately. In general, among all forecast factors, forecast factors such as hur850, hus 850 , ua500 and va500 show significant positive correlation with precipitation at most stations. The effect of slp, uas, and vas on precipitation was relatively weak and differed significantly among stations. In the wet node, the variables that contribute more to precipitation are often at high values and are prone to precipitation formation, and conversely, in the dry node, the variables that are more correlated with precipitation are often at low values and are not prone to precipitation formation.

(d) In order to test the applicability of this precipitation downscaling model in the North China region, the downscaling results were analyzed and evaluated in three aspects, including precipitation amount, precipitation frequency and extreme precipitation. All of the tested indicators performed well.

- 1) In the simulation of probability density function, the simulation results of its probability density curve are in good agreement with the observed values. The values of the indicators $B S$ are between 0 and $1.5 \times 10^{-4}$, and the values of the indicators Sscore are between 0.8 and 1 . Precipitation indicators Prtot and SDII can obtain better simulation results in summer: the average size of the simulation error of the precipitation downscale total precipitation (Prtot) is $-25.3 \mathrm{~mm}$ (the average error percentage is $-7.4 \%$ ). Precipitation intensity (SDII) shows negative simulation errors at most stations, with an average error size of $-1.1 \mathrm{~mm}$ (the average error percentage is $-11.6 \%$ ).

- 2) In general, the downscaling results are able to reproduce the frequency characteristics of 
the observed values. The total number of rainy days is underestimated to different degrees at all stations. The simulated error of the total number of rainy days (nr001) is -3.1days, and the SOMSD model slightly underestimates the total number of rainy days (error percentage $-9.8 \%$ ).

- 3) In the simulation of extreme precipitation characteristics, the downscaling model is more capable of simulating extreme summer precipitation events in North China, and the extreme precipitation contribution (P95T) is overestimated with an error of 3.4\% (error percentage 6.1\%). The maximum continuous rain days (CWD) at each station is underestimated by 1.1 days on average (error percentage $-20.0 \%$ ), and the maximum continuous rain-free days (CDD) is overestimated by 3.5 days on average (error percentage 31.1\%).

The SOM-SD model can simulate the probabilistic statistical characteristics of daily precipitation in North China, and the downscaling results are good in terms of precipitation amount, precipitation frequency and extreme precipitation. The model has good adaptability to North China and can be used for downscaling of current precipitation and scenario prediction of future precipitation in North China.

\section{Acknowledgments}

We acknowledge the National Centers for Environmental Prediction (NCEP) for the NCEP reanalysis data (http://www.cdc.noaa.gov). We acknowledge the helpful comments of three anonymous reviewers and the editor, who helped to improve this manuscript.

\section{Data Availability}

The codes and datasets generated during and/or analysed during the current study are available from the corresponding author on reasonable request. The NCEP dataset is available at http://www.cdc.noaa.gov.

\section{Declarations}

Conflict of interest The authors declare that there is no conflict of interest.

\section{References}

Brier GW (1950) Verification of forecasts expressed in terms of probability. Mon Weather Rev 78(1):1-3.

Burton A, Fowler H J, Blenkinsop S, et al. (2010) Downscaling transient climate change using a Neyman-Scott Rectangular Pulses stochastic rainfall model. J Hydrol 381(1): 18-32.

Cassano EN, Lynch AH, Cassano JJ, et al. (2005) Classification of synoptic patterns in the western Arctic associated with extreme events at Barrow, Alaska, USA. Clim Res 30(2): 83. 
Cassano EN, Lynch AH, Cassano JJ, et al. (2006) Classification of synoptic patterns in the western Arctic associated with extreme events at Barrow, Alaska. Clim Res 30: 83-97.

Cavazos T (1999) Large-scale circulation anomalies conducive to extreme precipitation events and derivation of daily rainfall in northeastern Mexico and southeastern Texas. J Clim 12(5): 1506-1523.

Chen L, Li W, Zhang P, Wang J (2003) Application of a new downscaling model to monthly precipitation forecast. quarterly journal of applied meteorology (6): 648-655.

Fowler HJ, Kilsby CG, Stunell J (2007) Modelling the impacts of projected future climate change on water resources in north-west England. Hydrol Earth Syst Sc 11(3): 1115-1126.

Fowler HJ, Kilsby CG, Stunell J (2007) Modelling the impacts of projected future climate change on water resources in north-west England. Hydrol Earth Syst Sc 11(3): 1115-1126.

Fu G, Charles S P, Yu J, et al. (2009) Decadal climatic variability, trends, and future scenarios for the North China Plain. J Clim 22(8): 2111-2123.

Hewitson BC, Crane RG (2002) Self-organizing maps: applications to synoptic climatology. Clim Res 22(1): 13-26. Hewitson BC, Crane RG (2006) Consensus between GCM climate change projections with empirical downscaling: precipitation downscaling over South Africa. Int J Climatol 26(10): 1315-1337.

Jia X, Chen L, Li W, Chen D (2010) Statistical downscaling based on BP-CCA: Predictability and application to the winter temperature and precipitation in China. Acta Meteorologica Sinica (3): 398-410.

Linderson M, Achberger C, Chen D (2004) Statistical downscaling and scenario construction of precipitation in Scania, southern Sweden. Nordic Hydrology 35(3): 261-278.

Liu W (2013) Improvements and implications of statistical downscaline models for daily rainfall, Institute of Geographic Sciences and Natural Resource Research, Chinese Academy of Sciences.

Lynch A, Uotila P, Cassano JJ (2006) Changes in synoptic weather patterns in the polar regions in the twentieth and twenty-first centuries, part 2: Antarctic. Int J Climatol 26(9): 1181-119.

Maraun D, Rust HW, Osborn TJ (2010) Synoptic airflow and UK daily precipitation extremes. Extremes 13(2): 133153.

Maraun D, Wetterhall F, Ireson AM, et al. (2010) Precipitation downscaling under climate change: Recent developments to bridge the gap between dynamical models and the end user. Rev Geophys 48(3).

Ning L, Mann M E, Crane R, et al. (2012) Probabilistic projections of climate change for the mid-Atlantic region of the United States: Validation of precipitation downscaling during the historical era. J Clim 25(2): 509-526. 

Journal of climate J Clim 20(17): 4356-4376.

690 Spak S, Holloway T, Lynn B, et al. (2007) A comparison of statistical and dynamical downscaling for surface temperature in North America. J Geophys Res-Atmos 112(D8).

692 Wang H, Fan K (2013) Recent changes in the East Asian monsoon. Chinese Journal of Atmospheric Sciences (in Chinese) 37 (2): 313-318.

Watterson IG (2008) Calculation of probability density functions for temperature and precipitation change under global warming. J Geophys Res 113(113):259-269.

Wei F, Huang J (2010) A study of downscaling factors of atmospheric circulations in the prediction model of summer precipitation in Eastern China. Chinese Journal of Atmospheric Sciences 34(1): 202-212.

Wilby RL, Charles SP, Zorita E, et al. (2004) Guidelines for use of climate scenarios developed from statistical downscaling methods. 1-27. Journal of Beijing Normal University (Natural Science) (5): 531-536. 